\title{
SISTEMA DE BIOTELEMETRÍA PARA ADQUISICIÓN DE SEÑALES BASADO EN BLUETOOTH
}

Pintos, Ramiro; Lombardero, Oscar G. $\left(^{*}\right)$

\section{Resumen}

El electrocardiograma permite visualizar la actividad eléctrica del corazón por medio de electrodos de superficie, los cuales captan las diferencias de potencial que ocurren en cada uno de los latidos. Es una de las herramientas más utilizada en la detección y el diagnóstico de enfermedades cardiovasculares, que hoy la tecnología permite hacerlo en forma digital, ahorrando tiempo y costo. El presente trabajo trata sobre el diseño y la implementación de un sistema de adquisición de señales cardíacas, empleando un dispositivo que registra el ECG, mediante un proceso de amplificación y filtrado y posteriormente lo envía a una PC mediante tecnología bluetooth, para visualizarla y almacenarla.

Dado el bajo costo de los elementos y la confiabilidad del equipo se consiguen un instrumento capaz de suplir algunas de las prestaciones de un electrocardiógrafo profesional.

\section{Introducción}

La electrocardiografía es una de las herramientas más utilizada en la detección y el diagnóstico de enfermedades cardiovasculares, y debido a los adelantos tecnológicos el registro electrocardiográfico ha pasado de la impresión de papel a la di- gitalización gracias al uso de las computadoras, dejando las posibilidades de realizar el análisis en un menor tiempo. Durante el proceso de activación del corazón se crean diferencias de potencial y a través de los electrodos fluye una corriente eléctrica. El electrocardiograma es la visualización de las proyecciones del vector cardiaco según tres ejes espaciales.

Un electrocardiógrafo básico está compuesto de un par de electrodos activos y uno de referencia, por lo general de $\mathrm{Ag} / \mathrm{Cl} \mathrm{Ag}$, colocados de tal manera que se pueda conformar el conocido triángulo de Einthoven. La combinación de los diferentes impulsos que se producen es recogido por sensores localizados al final de los electrodos y que son interpretados por medio de la señal que se envía y grafica en tiempo real, lo que da como resultado un ECG. La señal de ECG tiene un rango de frecuencia entre 0.05$150 \mathrm{~Hz}$ y los valores de la señal sobre la piel oscilan en pocos milivoltios. La interpretación adecuada del trazado electrocardiográfico permite el diagnóstico de los trastornos cardíacos.

\section{Diseño del Prototipo}

La Figura 1 muestra el diagrama de bloques del circuito completo, el cual consta de: 


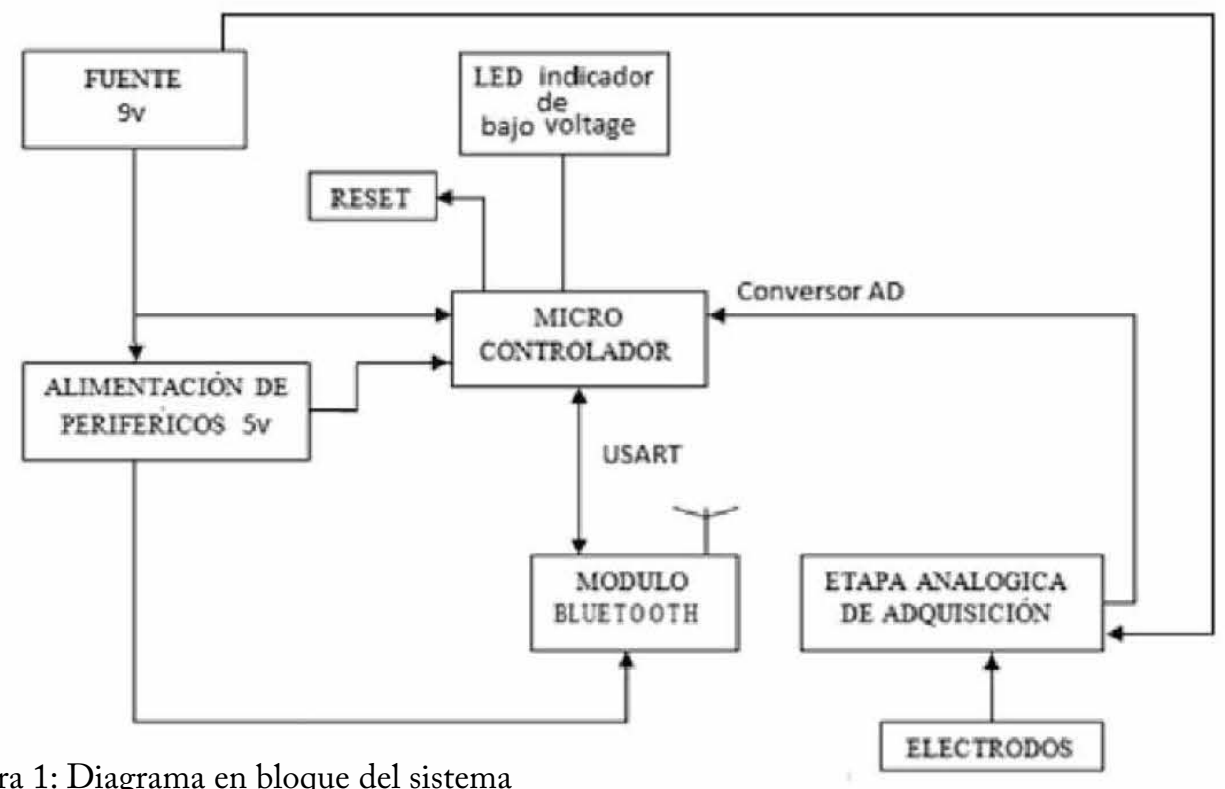

Figura 1: Diagrama en bloque del sistema

- Un núcleo central basado en un microcontrolador PIC que se comunica por medio de una interfaz de comunicación serie USART con el módulo bluetooth $\mathrm{HC} 05$.

- Una etapa de adquisición analógica conectada directamente a las entradas del convertidor analógico digital del micro para la posterior análisis de la señal adquirida.

- Una batería de 9V de donde se obtienen los $5 \mathrm{~V}$ por medio de un regulador de tensión LM7805.

- Un reset general para llevar al sistema a un estado de reinicio en caso de error.

- Un LED que nos señala el nivel de la batería, para un eventual recambio.

Para incrementar la autonomía, se utilizaron componentes de bajo consumo. Todos los circuitos integrados de la placa han sido seleccionados para trabajar a $5 \mathrm{~V}$ incluso el módulo bluetooth HC-05. Para obtener dicha tensión el sistema se alimenta con una batería de $9 \mathrm{~V}$.
Para la etapa de amplificación se utilizó un amplificador de instrumentación AD620 que tiene un alto rechazo en modo común (CMRR > 100db) y una alta impedancia de entrada, y una ganancia programada de 10.

La ecuación de ganancia para el AD620 está dada por:

$$
G=\frac{49,4 k \Omega}{R_{G}}+1
$$

por lo que para una ganancia de 10 el valor de será

$$
R_{G}=\frac{49,4 k \Omega}{G-1}=\frac{49,4 k \Omega}{10-1}=5,488 k \Omega
$$

Por lo tanto elegimos un valor de $\mathrm{Rg}$ normalizado de $5,6 \mathrm{k} \Omega$

Adicionalmente se incorporó un circuito de retroalimentación o driver de pierna derecha propuesto por el fabricante, para minimizar el ruido de línea, ya que pueden encontrarse problemas de desbalance 
en la entrada diferencial del amplificador de instrumentación. Para ello se utilizó el TL084 que posee cuatro amplificadores operacionales. Se colocaron dos resistencias de $24 \mathrm{k} \Omega$ para obtener un punto de referencia simétrico para el amplificador de pierna derecha y el valor del capacitor $\mathrm{C} 7$ fue seleccionado para mantener la estabilidad de la señal sobre la pierna derecha.

De igual forma se usó un A.O. en configuración integrador para disminuir el voltaje de offset a la salida del amplificador de instrumentación para evitar que el dispositivo entre en saturación. Con el filtro pasa bajo en el bucle de realimentación, la función transferencia del amplificador diferencial asume características de pasa alto. Por lo tanto para calcular los valores de $\mathrm{R} 1$ y $\mathrm{C} 1$ utilizamos la siguiente fórmula:

$$
f_{0}=\frac{1}{2 \pi R_{1} C_{1}}
$$

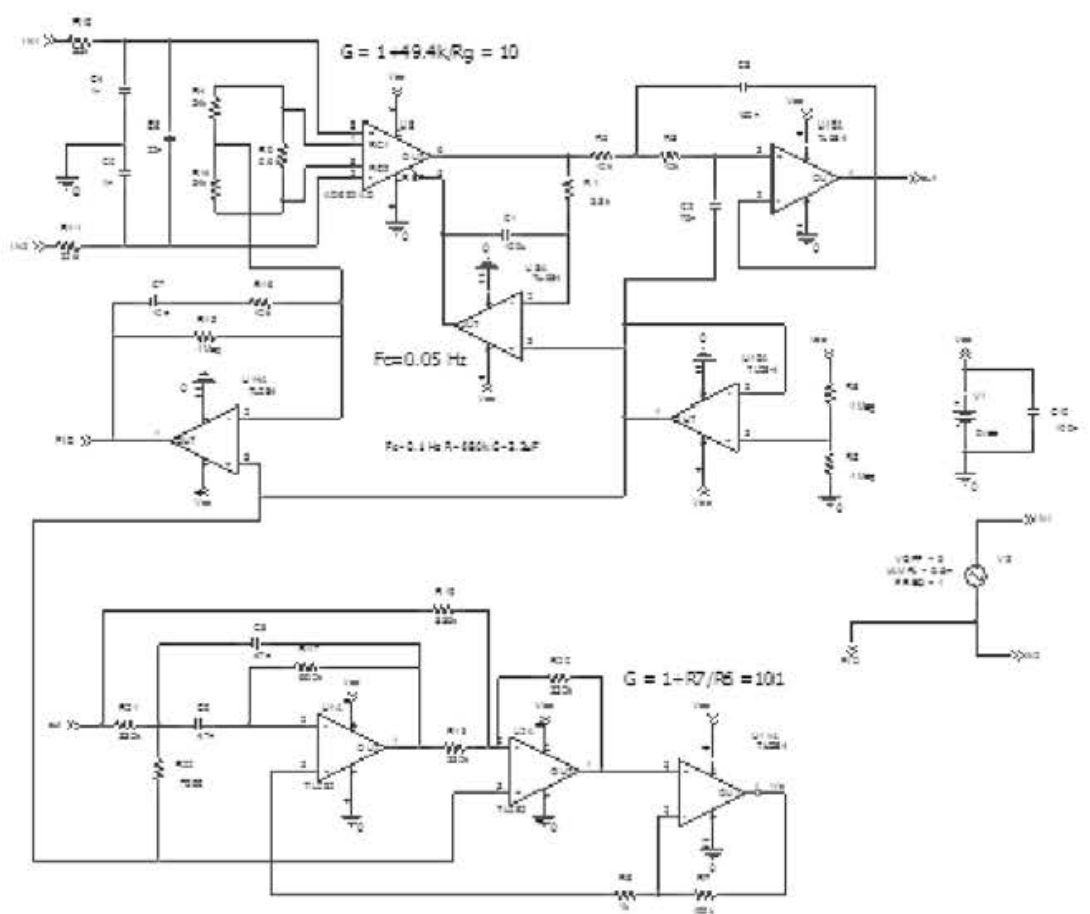

Figura 2: Esquema del sistema de adquisición
Fijamos el valor de $\mathrm{C} 1=100 \mathrm{uF}$ y obtenemos el valor de $\mathrm{R} 1=33 \mathrm{k} \Omega$ por lo que la fo $=0,05 \mathrm{~Hz}$ que va a ser la frecuencia de corte del pasa alto.

El acondicionamiento de la señal comprende una etapa de filtro activo el cual está comprendido por un filtro pasabajo de segundo orden Sallen Key cuya frecuencia de corte es de $\mathrm{fc}=150 \mathrm{hz}$ ya que la señal de ECG posee componentes de hasta esa frecuencia. Se eligió usar un filtro de Butterworth debido a que presenta una respuesta de la banda pasante plana y su pendiente desciende aceptablemente rápido al acercarse a la frecuencia de corte.

La última parte de la etapa de adquisición está compuesta por un amplificador no inversor el cual tiene una ganancia de 100 para darle una ganancia total al dispositivo de 1000. El esquema del circuito utilizado se lo puede apreciar en la Figura 2. 
La interfaz gráfica realizada en el GUI de MATLAB nos permite visualizar la señal electrocardiográfica en una ventana, a la vez que son almacenados en un archivo para su posterior análisis y poder realizar un registro con ella para el paciente.

Se realizaron dos plaquetas, una de ellas es la parte de adquisición de la señal y la otra plaqueta contiene el microcontrolador y el regulador de tensión. En la Figura 3 s epuede observar el dipositivo terminado. Se colocaron las dos plaquetas dentro de una caja plástica, llevando incorporado en la parte posterior una pinza de manera que pueda ser llevado cómodamente por el paciente, además del interruptor para encendido y apagado del mismo.

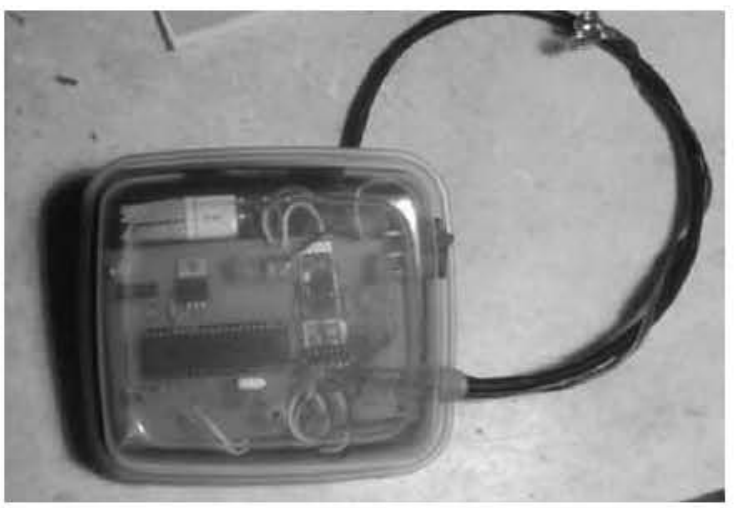

Figura 3: Vista frontal del registrador

\section{Ensayos y Resultados}

Se verificó el correcto funcionamiento del dispositivo, ubicando los electrodos de la siguiente manera:

- Pectoral izquierdo a la entrada no inversora del AD620.

- Pectoral derecho a la entrada inversora del AD620.

y el electrodo de referencia lo ubicamos a $10 \mathrm{~cm}$ del ombligo hacia la parte derecha del abdomen. Por último obtenemos la visualización de la señal en la PC como se observa en la Figura 4.

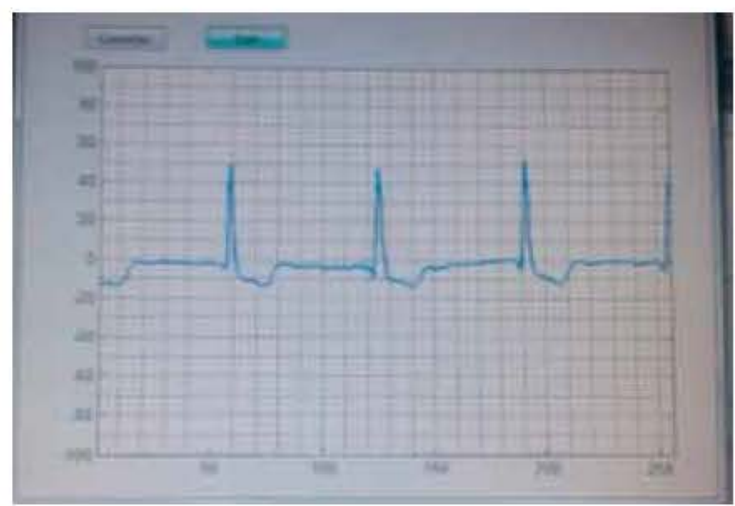

Figura 4: Visualización de la señal ECG en la interfaz gráfica

\section{Bibliografía}

[1] Compilador C y simulador Proteus para Microcontroladores, Eduardo García Breijo. Alfaomega Gr. Editor S.A. Mexico, 2008.

[2] Arthur C. Guyton Fisiología Humana Médica. Mc Graw Hill Interamericana Mexico, D.F. 2001, 10a Edición

[3] Robert 1. Boylestad, Louis Nashelsky. Electrónica: teoría de circuitos y dispositivos electrónicos, Pearson Prentice Hall, México, 2003. Pag. 798

[4] A. Bruce Carlson Circuitos. Ingeniería, Conceptos y Análisis de Circuitos Eléctricos Lineales Thomson Learning, México, D.F. 2001. 3a edición.

[5] Webster JG, Medical Instrumentation: Appli cation and design. Third Edition. Jhon Wiley \& Sons, New York, 1995.

[6] M.J. Moron, and R. Luque,. "Analysis of Bluetooth Transmission Delay

in Personal Area Networks". 2008 IEEE.

[7] A. M. Nasrabadi and M. H. Kani, "Design of ECG acquisition and transmission via Bluetooth with heart disease diagnosis". 2011 IEEE.

[8] Park and Cho, "Transmission of ECG Data with the Patch-Yype ECG sensor System using Bluetooth Low Energy". 2013 IEEE.

[9] Pandya and Desai, "A Novel algorithm for Bluetooth ECG”, IEEE Transactions on Biomedical Engineering, Vol. 59, No 11, November 2012. 